\title{
Using Two-Photon Intravital Imaging to Study Developmental Plasticity of Neural Circuits
}

Martin Munz, Delphine Gobert, Valerie Higenell, Marion R. Van Horn, Stephen Glasgow, Anne Schohl, and Edward S. Ruthazer

Montreal Neurological Institute, McGill University, Montreal, QC, CANADA H3A 2B4

Laser-scanning two-photon microscopy offers well-known advantages for imaging deeper into living tissue, but even in relatively translucent organisms like the zebrafish or albino Xenopus tadpole it can be a powerful tool for studying brain development [1]. The visual system in the Xenopus tadpole utilizes both molecular guidance and activity-dependent cues to form appropriate connections [2]. N-methyl-Daspartate (NMDA) type glutatmate receptors have been shown to participate in the activity-dependent mechanisms of circuit formation, however their specific contributions to the process of axonal projection structural refinement is not fully understood.

We took advantage of the fact that the infrared light used for $2 \mathrm{P}$ excitation does not drive visual responses in the tadpole eye, allowing us to characterize the effects in real time of patterned visual stimuli on retinotectal axon remodeling in vivo, revealing a role for NMDA receptors as correlation detectors. In addition, to analyze concomitant changes in synaptic connectivity, we used the non-linear nature of $2 \mathrm{P}$ excitation to identify synaptically coupled pairs of neurons in vivo by $3 \mathrm{D}$ photoactivation of PA-GFP for perforated patch electrophysiology. Finally we used morpholino antisense knockdown of the NR1 subunit of NMDA receptors specifically targeted to the RGCs in order to assess whether presynaptic NMDA receptors might contribute a role in for the growth and branching of GFP labeled RGC axons in the developing circuit that is distinct from the role played by postsynaptic NMDA receptors. Our results provide novel insights into how neural activity guides the remodeling of axons in the developing brain and show that the NMDARs in pre and postsynaptic cells perform opposing functions for circuit formation with the former promoting branch formation and the latter limiting it (Figure 1).

\section{References:}

[1] Ruthazer, E.S. and Cline, H.T., Journal of Real-Time Imaging: Special Issue on Imaging in Bioinformatics 8 (2002), p. 175.

[2] Ruthazer, E.S. and Cline, H.T., Journal of Neurobiology, 59 (2004), p. 134. 
Figure 1: A unified model for NMDA receptor function in the developing retinotectal circuit. A) Presynaptic retinal ganglion cell axons terminate on postsynaptic targets. Cells that are near neighbors in the eye have similar firing patterns (green and blue) whereas more distantly separated cells fire with uncorrelated patterns (green and red). B) Axons that fire in synchrony stabilize their existing connections and axon branches over time whereas axons that fire asynchronously destabilize and add many additional exploratory branches, eventually finding their way to the better matched partner. Postsynaptic NMDARs mediate the stabilization of branches whereas the presynaptic NMDARs may contribute to exploratory or constitutive branching.

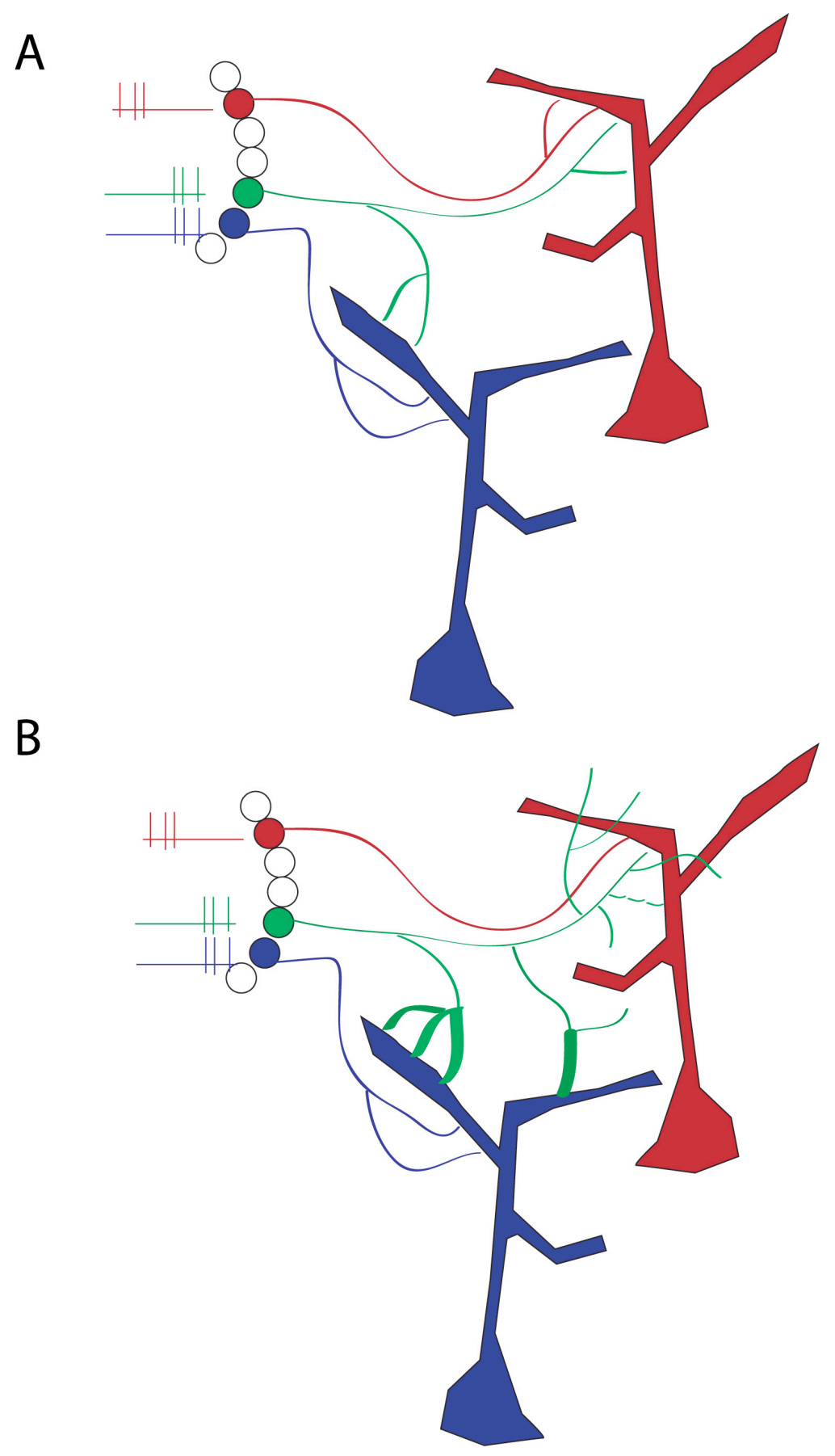

